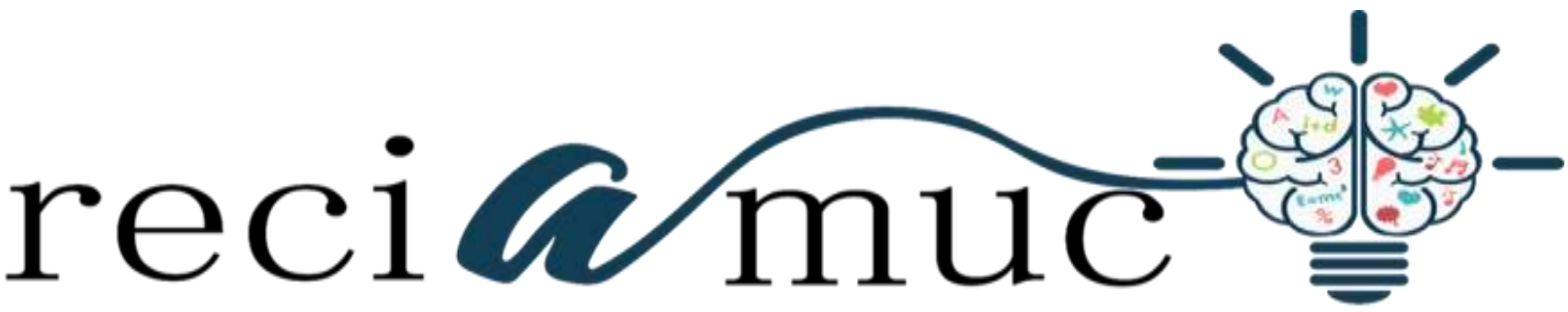 \\ Revista cientifica de investigación actualización del mundo de las ciencias
}

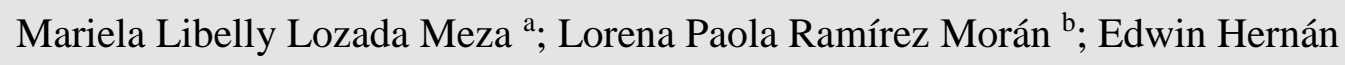
Alvarado Chicaíza ${ }^{c}$; Carol Johana Cajas Bejarano ${ }^{\text {d }}$

Evaluación del estado nutricional de gestantes universitarias, UNEMI 2018: resultados del plan piloto

Evaluation of the nutritional status of university gestants, UNEMI 2018: results of the pilot plan

Revista Científica de Investigación actualización del mundo de las Ciencias. Vol. 3 núm., 1, enero, ISSN: 2588-0748, 2018, pp. 483-516

DOI: $10.26820 / \mathrm{reciamuc/3.(1).enero.2019.483-516}$

URL: $\underline{\text { http://reciamuc.com/index.php/RECIAMUC/article/view/246 }}$

Código UNESCO: 3206 Ciencias de la Nutrición

Tipo de Investigación: Artículo de Revisión

\author{
Editorial Saberes del Conocimiento
}

Recibido: 10/12/2018

Aceptado: 20/01/2019

Publicado: 30/01/2019

Correspondencia: mlozadam@unemi.edu.ec

a. Especialista en Nutrición Deportiva; Doctora en Nutrición y Dietética; Universidad Estatal de Milagro; Milagro, Ecuador; mlozadam@unemi.edu.ec

b. Master of Public Health; Bachelor of Health Services Administration; Universidad Estatal de Milagro; Milagro, Ecuador; lorenap1821@gmail.com

c. Licenciado en Enfermería; Universidad Estatal de Milagro; Milagro, Ecuador; edher_alvarado@hotmail.com

d. Licenciada en Enfermería; Universidad Estatal de Milagro; Milagro, Ecuador; caroljohanabe@ hotmail.com 


\section{Evaluación del estado nutricional de gestantes universitarias, UNEMI 2018: resultados del plan piloto}

Vol. 3, núm. 1., (2019)

Mariela Libelly Lozada Meza; Lorena Paola Ramírez Morán; Edwin Hernán Alvarado Chicaíza; Carol Johana Cajas Bejarano

\section{RESUMEN}

Introducción: La evaluación del estado nutricional en gestantes es un factor determinante informativo en el desarrollo fetal y en el peso del recién nacido; mujeres con normopeso pregestacional hará una ganancia de 9 a $11 \mathrm{~kg}$. Objetivo. Determinar el estado nutricional de las estudiantes gestantes en la Universidad estatal de Milagro, con instrumentos validados por expertos en salud Método: El diseño de investigación fue cuantitativa, bibliográfica-documental, retrospectiva, descriptiva de cohorte transversal. La población fue de 174 estudiantes, la muestra 81 gestantes, aplicando un plan piloto a 20 en octubre 2018, como instrumentos se elaboró dos encuestas con 5 secciones y 14 ítems, validado por juicios de expertos en salud-nutrición, comprobando validez, pertinencia y coherencia, los resultados son de interés en la salud pública. Resultados: el 65\% de gestantes proceden de otros cantones, un 60\% está en unión libre, el 90\% con soporte económico de la pareja y conviven con la misma, el 20\% son adolescentes. Según el IMC/Eg el 5\% de gestantes esta en bajo peso, $40 \%$ peso normal, $45 \%$ sobrepeso y $10 \%$ obesidad; el 35\% dijo presentar anemia. En el consumo alimentario, el 65\% realizan 4 a 5 comidas/día, consideran una dieta variada $75 \%$, el cálculo nutrimental del consumo en r24hrs muestra que el $80 \%$ tiene una dieta deficiente, solo el $20 \%$ es adecuado. Conclusión: la evaluación del estado nutricional a través de los métodos dietético y antropométrico establece el criterio diagnóstico de salud de las gestantes universitarias con condiciones de mal nutrición al déficit y exceso.

Palabras Claves: Gestante; Estado nutricional; Consumo alimentario; Índice de Masa Corporal. 


\section{Evaluación del estado nutricional de gestantes universitarias, UNEMI 2018:}

resultados del plan piloto

Vol. 3, núm. 1., (2019)

Mariela Libelly Lozada Meza; Lorena Paola Ramírez Morán; Edwin Hernán Alvarado Chicaíza;

Carol Johana Cajas Bejarano

\section{ABSTRACT}

Introduction: The evaluation of the nutritional status in pregnant women is an informative determining factor in the fetal development and in the weight of the newborn; Women with prepregnancy normal weight will make a gain of 9 to $11 \mathrm{~kg}$. Objective. To determine the nutritional status of pregnant students at the State University of Milagro, with instruments validated by health experts. Method: The research design was quantitative, bibliographic-documentary, retrospective, descriptive of the transversal cohort. The population was 174 students, 81 pregnant women sample, applying a pilot plan to 20 in October 2018, as instruments were developed two surveys with 5 sections and 14 items, validated by expert judgments in health-nutrition, verifying validity, relevance and coherence, the results are of interest in public health. Results: $65 \%$ of pregnant women come from other cantons, $60 \%$ are in free union, $90 \%$ with economic support of the couple and live with it, $20 \%$ are adolescents. According to the IMC / Eg, 5\% of pregnant women are in low weight, $40 \%$ normal weight, $45 \%$ overweight and $10 \%$ obese; $35 \%$ said they present anemia. In food consumption, $65 \%$ carry out 4 to 5 meals / day, consider a $75 \%$ varied diet, the nutritional calculation of consumption in $\mathrm{r} 24 \mathrm{hrs}$ shows that $80 \%$ have a deficient diet, only $20 \%$ is adequate. Conclusion: the evaluation of the nutritional status through the dietetic and anthropometric methods establishes the diagnostic criteria of health of the university pregnant women with conditions of bad nutrition to the deficit and excess.

Key Words: Pregnant; Nutritional status; Food consumption; Body Mass Index. 


\section{Evaluación del estado nutricional de gestantes universitarias, UNEMI 2018: resultados del plan piloto}

Vol. 3, núm. 1., (2019)

Mariela Libelly Lozada Meza; Lorena Paola Ramírez Morán; Edwin Hernán Alvarado Chicaíza; Carol Johana Cajas Bejarano

\section{Introducción.}

El estado nutricional como indicador en la valoración de la gestante identifica la condición de salud de la madre y el bebé, para ello la evaluación nutricional es un método que permite determinar si se cumplen con eficacia las necesidades nutricionales en esta etapa. Se considera que el peso al nacer guarda paralelismo con la ganancia de peso materno, sin embargo, la desnutrición materna por la ganancia ponderal escasa durante el embarazo, así como el sobrepeso y obesidad son factores de riesgo reconocidos para el niño o niña; según la Organización mundial de la salud (OMS) para el 2014 estimaba que en los últimos 30 años el sobrepeso en el embarazo sería el doble, reconociendo esta situación como un conflicto para la salud pública, pues incrementa los riesgos obstétricos y neonatales.

La ganancia de peso durante el embarazo es uno de los indicadores primarios que garantiza las mejores condiciones de salud y nutrición de la madre y el niño, son múltiples factores los que alteran este requisito; desde la ingesta inadecuada e insuficiente de alimentos y nutrientes, manifestaciones depresivas y trastornos emocionales que se refleja como fatiga, falta de concentración, insomnio e hipersomnia, pasando por alteraciones debidas a problemas familiares, laborales y/o conyugales, además del gasto energético que demanda el trabajo físico en jornadas laborales extenuantes (MSP, 2012).

Así mismo el adecuado diagnóstico y clasificación nutricional en la embarazada permite una mejor identificación de los riesgos asociados a desnutrición, obesidad materna y su correlación con enfermedades del embarazo entre ellas la diabetes gestacional, hipertensión y 


\section{Evaluación del estado nutricional de gestantes universitarias, UNEMI 2018:}

resultados del plan piloto

Vol. 3, núm. 1., (2019)

Mariela Libelly Lozada Meza; Lorena Paola Ramírez Morán; Edwin Hernán Alvarado Chicaíza;

Carol Johana Cajas Bejarano

preeclamsia, mientras que en el neonato el incremento del riesgo de bajo peso, distocias y muerte fetal (Grandi, Luchtenberg, \& Sola, 2007).

Por lo tanto una valoración de estado nutricional implica a más del registro de medidas antropométricas y consumo alimentario, las pruebas bioquímicas elementales como hemoglobina. La valoración nutricional puede ser definida como la interpretación de la información obtenida a partir de estudios antropométricos, alimentarios, bioquímicos y clínicos. Dicha información es utilizada para determinar el estado nutricional de individuos o grupos de población en la medida que son influenciados por el consumo y la utilización de nutrientes. (UBA, 2016)

Ya en el análisis de la situación de salud en gestantes en Latinoamérica el Sistema de Salud ha implementado cambios estructurales para reducir la muerte materna con una mejor respuesta en Paíces como Uruguay, Perù y Ecuador; en el País las primeras causas de mortalidad materna siguen siendo: hemorragia posparto $17.01 \%$, hipertensión gestacional $12.45 \%$ y eclampsia $12.86 \%$, las cuales están relacionadas o que se cree su origen es por deficiencias nutricionales, además se evidencian que la anemia es un factor de riesgo de muerte materna, por lo tanto, abordar la deficiencia de este mineral podría resultar sustancial en la reducción de las muertes maternas. (MSP, 2014)

Es decir que la identificación y registro de datos de la gestante tanto clínico como alimentario es responsabilidad de las unidades de salud, en la Universidad Estatal de Milagro (UNEMI) estas acciones se cumplirían a través de su Departamento de Bienestar Universitario que cuenta con un centro de atención en salud de primer nivel, el mismo es el más cercano a la 


\section{Evaluación del estado nutricional de gestantes universitarias, UNEMI 2018: resultados del plan piloto}

Vol. 3, núm. 1., (2019)

Mariela Libelly Lozada Meza; Lorena Paola Ramírez Morán; Edwin Hernán Alvarado Chicaíza; Carol Johana Cajas Bejarano

población educativa y, es de su competencia facilitar y coordinar el flujo del usuario dentro del sistema, además que garantiza una referencia y contra referencia adecuada y asegurar la continuidad y longitudinalidad de la atención. El departamento cuenta con el personal profesional: médico. psicóloga, nutricionista, odontólogo, enfermera y auxiliar de enfermería y trabajadora social.

\section{Resultados.}

La población de estudio conformado por estudiantes en periodo de gestación en un $20 \%$ aun adolescentes, $75 \%$ adulto joven, procedentes de cantones diversos, con un estado civil en unión libre de un 60\%, de las mismas el 90\% se encuentran en dependencia económica de sus parejas. En lo referente al estado nutricional el $60 \%$ presenta algún grado de malnutrición sea al sobrepeso y obesidad en un 50\%, así como bajo peso en el 5\% de las mismas; el recordatorio de la gestante identifico que al menos el 35\% fue advertida que tenía anemia. Además el análisis de consumo alimentario identifico hábitos como el desayuno en el 65\% con 45 tiempos de comida en 24 horas, mas sin embargo el cálculo de aporte nutrimental de la dieta determinó que $60 \%$ tenia una alimentación deficiente, con predominio en el consumo de cereales, tubérculos, grasas, azúcares.

\section{Método.}

En el desarrollo del proyecto de investigación la validación de los instrumentos de evaluación de estado nutricional es el paso previo, esto implica procesos como refiere Goni, Martínez, Santiago, \& Cuervo (2013), en su estudio donde resaltan que la valoración del estado 


\section{Evaluación del estado nutricional de gestantes universitarias, UNEMI 2018:}

resultados del plan piloto

Vol. 3, núm. 1., (2019)

Mariela Libelly Lozada Meza; Lorena Paola Ramírez Morán; Edwin Hernán Alvarado Chicaíza;

Carol Johana Cajas Bejarano

nutricional es de gran utilidad y esta se realiza mediante diversos parámetros, entre estos el más

utilizado es la toma de medidas antropométricas como el peso y la talla para el cálculo del Índice de Masa Corporal, a más de los datos de valoración nutricional complementarios. Además, sostienen que una adecuada intervención durante el embarazo ayuda a prevenir enfermedades tanto en la madre como en el bebé y así de esta manera se lograría mantener un adecuado estado de salud y nutrición, los cuales al verse alterados representan un problema de gran impacto en la salud pública. Entre otros estudios también se analiza la relación del estado nutricional con las condiciones socioeconómicas de embarazadas atendidas en un centro de salud La Concordia - Ecuador determina que existe una relación significativa entre la composición corporal y los aspectos socioeconómicos, (Torres, 2016).

En similitud el presente trabajo aplica métodos de valoración antrometrica, dietaría como el recordatorio de 24 horas y el cuestionario de frecuencia de consumo de alimentos que se utilizan con mayor frecuencia en estudios epidemiológicos por su confiabilidad y validez. Cabe mencionar que en esta prueba piloto todas las participantes fueron informadas sobre el tema de estudio y firmaron el consentimiento informado con la finalidad de garantizar el manejo confidencial de los datos y su anonimato, se consideran los datos de identificación e informativos generales de las gestantes participantes para establecer factores medibles relacionados al estado nutricional actual; los criterios citados son validados por 3 profesionales con títulos de PhD y 3 Técnicos Nutricionistas

En este estudio el tipo de metodología que se aplicó fue la investigación cuantitativa, para medir las variables cuantificables de estado nutricional con el cálculo de índice de masa 


\section{Evaluación del estado nutricional de gestantes universitarias, UNEMI 2018: resultados del plan piloto}

Vol. 3, núm. 1., (2019)

Mariela Libelly Lozada Meza; Lorena Paola Ramírez Morán; Edwin Hernán Alvarado Chicaíza; Carol Johana Cajas Bejarano

corporal según edad gestacional (IMC) y, consumo alimentario a través del conteo calórico por macronutrientes en la dieta consumida en r24hrs a más de la frecuencia de consumo por grupos de alimentos, según su contexto fue una investigación de campo, por su objetivo gnosiológico es descriptiva, de cohorte transversal el cual permitió constatar los hallazgos de este estudio en el tercer trimestre del 2018.

El universo estaba constituido 174 mujeres que se identificaron como gestantes a través del Sistema de gestión académica- aula virtual de las 3922 estudiantes femeninas matriculadas en el 2018; la población con respecto al estudio fue de 81 gestantes efectivas en algún periodo de gestación, aplicando un plan piloto de 20 estudiantes gestantes con edades entre 18 y 34 años, en la obtención de datos para los resultados analizados en este estudio se solicitó llenar la encuesta y una ficha de registro de datos de valoración antropométrica y dietaría, revisada por juicio de expertos en salud calificando validez, pertinencia y confiabilidad clasificando los ítems con sus respectivos objetivos, en el objetivo general que indica; determinar el estado nutricional de las gestantes universitarias a través del IMC/G y, calidad de dieta dada por el porcentaje de adecuación entre el consumo y el requerimiento calórico recomendado por trimestre de gestación. El puntaje con respecto a la validez fue de $98 \%$, en pertinencia fue un valor del $95 \%$, mientras que en confiabilidad un $93 \%$.

Por otro lado, los items del primer objetivo específico indica; Identificar datos informativos de la estudiante gestante, alcanzó un porcentaje de calificación para validez un 100\%, la pertinencia un $98 \%$ mientras que la confiabilidad fue de $98 \%$. Los items del segundo objetivo específico que establece determinar el patrón alimentario mediante el analisis de frecuencia de 


\section{Evaluación del estado nutricional de gestantes universitarias, UNEMI 2018: resultados del plan piloto}

Vol. 3, núm. 1., (2019) Mariela Libelly Lozada Meza; Lorena Paola Ramírez Morán; Edwin Hernán Alvarado Chicaíza; Carol Johana Cajas Bejarano

consumo alimentario en una semana, además de un registro de consumo por recordatorio reprotrospectivo de 24 horas previas a la aplicación de la encuesta, considerando además actitudes y prácticas alimentarias, fue calificado con un $96 \%$ de validez, $94 \%$ en pertinencia y 92\% en confiabilidad. Por último, se calificó los items en base al tercer objetivo específico; Analizar el índice de masa corporal para identificar riesgo de bajo peso, sobrepeso u obesidad la validez fue calificada con un promedio de porcentaje del $96 \%$, la pertinencia de $98 \%$, mientras que la confiabilidad fue de 96\%, recogiendo de manera segura los datos importantes que apelan a esta investigación.

La encuesta contiene en la variable datos informativos de la gestante: Nombres y apellidos; edad de la gestante categorizada por rangos (15 - 19 años), ( 20 - 24 años), (25 - 29 años), (30 - 34 años) ( $\geq 35$ años). Lugar de procedencia (A. Milagro, B. Otros cantones, C. Otra provincia). Estado civil:( A. Soltera, B. Casada, C. Unión libre, D. Divorciada, E. Viuda. Además, se consideró analizar la fuente de provisión económica, las alternativas fueron:( A. Padres, B. Autofinanciamiento, C. Pareja, D. Otros familiares, E Aportes de todos los anteriores). En cuanto a su convivencia actual: (A. Padres, B. Compañeros estudiantes, C. Esposo, D. Familiares, E. Sola). Además se ha incluido la edad gestacional en semanas y, por recordatorio si presenta anemia, no, o no recuerda. En la variable patrón alimentario se determina por el consumo de alimentos por preferencia, se propuso: frecuencia de consumo en 15 grupos alimentos en la semana clasificados como: Grupo 1- lácteos y derivados; Grupo 2huevos; Grupo 3- carnes y vísceras; Grupo 4- Embutidos; Grupo 5- pescados y mariscos; Grupos 6- leguminosas y oleaginosas; Grupo 7- semillas; Grupo 8- especias, Grupo 9- verduras 


\section{Evaluación del estado nutricional de gestantes universitarias, UNEMI 2018: resultados del plan piloto}

Vol. 3, núm. 1., (2019)

Mariela Libelly Lozada Meza; Lorena Paola Ramírez Morán; Edwin Hernán Alvarado Chicaíza; Carol Johana Cajas Bejarano

hortalizas y otros vegetales; Grupo 10- frutas; Grupo 11- tubérculos y raíces; Grupo 12cereales, harinas, pastas y otros derivados; Grupo 13- Azúcares; grupo 14- grasas; Grupo 15otros productos.

La valoración de la dieta consumida por la gestante se describe a través del cálculo y análisis de aporte nutrimental de calorías, macronutrientes y hierro en las preparaciones de un día en los 5 tiempos de comida (desayuno, media mañana, almuerzo, media tarde y merienda) y, registrando el tiempo, horario, preparación y/o alimento, cantidad en medida casera, cantidad en gramos o mililitros y método de cocción del alimento o preparación antes de la encuesta. El recordatorio de 24 horas de pasos múltiples el método seleccionado, elaborado por el Departamento de Agricultura de Estados Unidos (USDA, US Department of Agriculture), el cual es un método que consiste en interrogar al paciente sobre todo lo que ingirió el día de ayer, sólidos y líquidos. Se escogió este modelo por las amplias evidencias científicas que validan su confiabilidad, además que se considera uno de los que ayuda a minimizar el riesgo de reportes subestimados en la alimentación, por medio de este método se pretende ayudar al entrevistado a interesarse más en la entrevista y con eso, recordar todo lo consumido (Suverza \& Haua, 2010)

Para el análisis del aporte nutrimental se utilizó la tabla de composición de alimentos del Instituto de Nutrición de Centroamérica y Panamá (INCAP), considerada una de las de mayor fiabilidad y de aplicación por la amplia gama de productos y alimentos, así como el soporte técnico de ensayos bioquímicos de contenidos nutrimentales en los alimentos (INCAP, 2012). El cálculo considera el aporte en 100 gramos de muestra por alimento en cada preparación, valorando que el aporte por gramo de nutriente es de 4, 9 y 4 calorías para proteínas, grasas e 


\section{Evaluación del estado nutricional de gestantes universitarias, UNEMI 2018:}

resultados del plan piloto

Vol. 3, núm. 1., (2019)

Mariela Libelly Lozada Meza; Lorena Paola Ramírez Morán; Edwin Hernán Alvarado Chicaíza;

Carol Johana Cajas Bejarano

hidratos de carbono respectivamente, se procedió a realizar la sumatoria por aporte en alimentos para conseguir el total de kilocalorías consumidas.

Las actitudes y prácticas alimentarias, recopila datos como hábitos de desayuno en las gestantes categorizado como: (Desayuna siempre, A veces o Nunca), también se consideró la percepción de la participante de que la dieta que consume es o no la adecuada para la edad gestacional en la cual respondían (Sí - No - No lo se). En cuanto a los tiempos de comida al día se indagó respecto al número de veces (Frecuencia de $\leq$ a 3, de 4 a 5 y $\geq$ a 6).

En cuanto al cálculo del requerimiento calórico se realizó considerando el peso, sexo, edad, y nivel de actividad física, adicionando las kilocalorías extras según estado nutricional y trimestre de embarazo establecido en la Guía de concepción del Ministerio de Salud Pública del Ecuador, el cual indica lo siguiente:

Carga calórica extra en la dieta de la gestante según estado nutricional

\begin{tabular}{llll}
\hline & $\begin{array}{l}\text { Primer } \\
\text { trimestre }\end{array}$ & $\begin{array}{l}\text { Segundo } \\
\text { trimestre }\end{array}$ & $\begin{array}{l}\text { Tercer } \\
\text { trimestre }\end{array}$ \\
\hline Bajo peso & $150 \mathrm{kcal}$ & $200 \mathrm{kcal}$ & $300 \mathrm{kcal}$ \\
Peso normal & & $350 \mathrm{kcal}$ & $450 \mathrm{kcal}$ \\
Sobrepeso/Obes & & $350 \mathrm{kcal}$ & $350 \mathrm{kcal}$ \\
\hline
\end{tabular}

Fuente: Recuperado de Guía Maternidad y Primera Infancia (MSP, 2017). Pág 28

Finalmente, para calcular el porcentaje de adecuación de la dieta se dividió el total de kilocalorías consumidas para el total de kilocalorías recomendadas por cien, en el cual se interpretó que de 90 a 110\% clasifica como dieta adecuada, < del 90\% dieta deficiente y > del 


\section{Evaluación del estado nutricional de gestantes universitarias, UNEMI 2018: resultados del plan piloto}

Vol. 3, núm. 1., (2019)

Mariela Libelly Lozada Meza; Lorena Paola Ramírez Morán; Edwin Hernán Alvarado Chicaíza; Carol Johana Cajas Bejarano

$110 \%$ dieta excesiva. De esta manera, se logró obtener los resultados necesarios para la validación del instrumento que se empleará en la evaluación del estado nutricional en estudiantes gestantes.

\section{Método dietario}

Castell, Serra resume que la dieta, junto con los estilos de vida, es un importante factor determinante del estado de salud del individuo y de la comunidad. La valoración de la ingesta dietética a nivel poblacional aporta información básica para conocer la frecuencia y la distribución de posibles desequilibrios dietéticos y/o nutricionales, así como para orientar el diseño de políticas nutricionales dirigidas a mejorar los hábitos alimentarios y los niveles de salud de una comunidad, revisa las características, ventajas y limitaciones del método de recordatorio dietético de $24 \mathrm{~h}(\mathrm{RD} 24 \mathrm{~h})$, uno de los métodos más ampliamente utilizados en epidemiologia nutricional para determinar ingesta de alimentos, energía y nutrientes, en encuestas nutricionales a nivel nacional, en estudios transversales, ensayos clínicos, estudios de cohortes y también en valoraciones individuales y en evaluación de dieta total. Se destaca la importancia, para reducir los principales puntos de sesgo, de la formación de los encuestadores, las herramientas de apoyo y las aportaciones de las nuevas tecnologías.

Consiste en especificar y cuantificar todos los alimentos y bebidas consumido en un periodo de tiempo anterior a la entrevista de 24 horas, desde la primera ingesta de media noche hasta la última ingesta de media noche siguiente. Es una técnica que consiste en interrogar al encuestado acerca de cada uno de los alimentos y bebidas consumidos el día anterior. Estima el consumo de alimentos y su traducción a unidades de energía y nutrientes, determina los 


\section{Evaluación del estado nutricional de gestantes universitarias, UNEMI 2018:}

resultados del plan piloto

Vol. 3, núm. 1., (2019)

Mariela Libelly Lozada Meza; Lorena Paola Ramírez Morán; Edwin Hernán Alvarado Chicaíza;

Carol Johana Cajas Bejarano

promedios de ingesta. El registro lo hace la misma persona y puede ser realizado en un día o en múltiples días, para verificar la confiabilidad de la información obtenida. Las desventajas del método son que tiende a subvalorar la ingesta de alimentos, se necesitan varios días para obtener datos confiables, requiere de entrevistadores expertos y es difícil calcular el tamaño de las porciones. Entre las ventajas, se trata de una encuesta de rápida realización, ya que toma de 15 a 20 minutos, se basa únicamente en la memoria, no se modifican los patrones alimentarios del entrevistado y el encuestado no necesita saber leer ni escribir.

Entre las recomendaciones para optimizar la recogida de los datos se considera: anotar con la mayor precisión posible todos los alimentos y bebidas consumidos en las últimas 24 horas, empezar por el desayuno del día anterior y continuar hasta completar el recuerdo de la dieta del día entero, anotar los alimentos consumidos entre horas. Además, se debe registrar la calidad del alimento (leche entera o desnatada, pan blanco o integral, tipo de carne). Es importante saber estimar la cantidad consumida medidas caseras o en raciones (grande, mediana, pequeña). Considerar si empleo grasa (manteca o aceite) en las preparaciones culinarias, el pan, el azúcar o las bebidas consumidas. Registrar el método de preparación culinario (cocido, frito, asado). Finalmente, Para facilitar el recuerdo, escribir inicialmente el menú consumido en cada comida y luego describir detalladamente los ingredientes. Igualmente, para ayudar a la memoria, es muy práctico recordar dónde comimos, con quién, a qué hora, quién preparó la comida.

En cuanto a los determinantes de la calidad del recordatorio de 24 horas, la información obtenida dependerá básicamente de cinco factores; el sujeto entrevistado, el entrevistador, la 


\section{Evaluación del estado nutricional de gestantes universitarias, UNEMI 2018: resultados del plan piloto}

Vol. 3, núm. 1., (2019)

Mariela Libelly Lozada Meza; Lorena Paola Ramírez Morán; Edwin Hernán Alvarado Chicaíza; Carol Johana Cajas Bejarano

cuantificación de la ración, la codificación del recordatorio y las tablas de composición. Los sujetos que interviene en este tipo de estudio son de interés así:

- Sujeto entrevistado: Debe recordar de forma precisa el consumo de alimentos del día anterior, esto viene influenciado por la edad, el sexo, el nivel de educación. Tanto los niños como las personas mayores tienen dificultad para estimar de forma adecuada el consumo de alimentos, los detalles y la descripción de los mismos, para evitar errores se deberá hacer en presencia de la madre o de la persona responsable de su cuidado. En general se estima que la mujer recuerda mejor lo que come que el hombre, además los individuos con un nivel de educación superior son capaces de describir con más exactitud su alimentación. La estimación de las cantidades también se puede falsear de una forma más o menos consciente al ocultar las cantidades reales o bien el consumo de dulces.

- Encuestador: Es preciso un aprendizaje previo. Técnicas generales de presentación y entrevista, técnicas de recordatorio de ingesta dietética: volúmenes, peso en crudo o cocido, limpio, bruto. Deben conocer los hábitos y costumbres de la comunidad encuestada. La estandarización de los encuestadores es necesaria, así como que nunca debe dar opiniones al encuestado sobre consumo, o hábitos convenientes o alimentos perjudiciales.

- Cuantificación de la ración: Se pretende estimar la ración precisa de un alimento determinado, consumido el día anterior y que no tiene por qué coincidir con la ración habitual. Los errores derivados de la imprecisión de la medición junto con los inherentes a las tablas de composición de alimentos son las principales fuentes de error. Es 


\section{Evaluación del estado nutricional de gestantes universitarias, UNEMI 2018:}

resultados del plan piloto

Vol. 3, núm. 1., (2019)

Mariela Libelly Lozada Meza; Lorena Paola Ramírez Morán; Edwin Hernán Alvarado Chicaíza;

Carol Johana Cajas Bejarano

conveniente pedir al encuestado que especifique su ración mediante medidas caseras o a través de comparación con distintos modelos.

- Tablas de composición de los alimentos: Son necesarias para el estudio nutricional de grupos de población, pero suelen presentar limitaciones debidas en parte a la metodología analítica seguida y a la recopilación de datos reseñados en las mismas. Las tablas que habitualmente se utilizan se basan en datos recopilados a partir de referencias bibliográficas. Existen diversas tablas de composición de alimentos editadas en todo el mundo, y algunas específicas para cada país, adaptadas a las peculiaridades de cada región. Actualmente con las tablas de composición de alimentos utilizadas a través de programas informáticos más o menos sofisticados y que en esencia son una hoja de cálculo, se evita la pérdida de tiempo y cansancio en el cálculo. Pero los errores procedentes de tablas inadecuadas no se corregirán por el uso de un programa informático. El investigador deberá previamente seleccionar aquella base de datos que sea más fiable y más de acuerdo con el tipo de estudio a realizar.

\section{Método antropométrico}

Rached (2015) señala que el riesgo menor de complicaciones durante el embarazo o en el parto como consecuencia de una ganancia de peso adecuada de la gestante a lo largo de este período ha generado un creciente interés con relación a los efectos que tiene el estado nutricional materno sobre el producto de la gestación y el desarrollo infantil. La evaluación nutricional de la gestante comprende la evaluación inicial y el seguimiento. La evaluación inicial permite determinar el estado nutricional de la embarazada y estimar el riesgo probable de peso bajo al 


\section{Evaluación del estado nutricional de gestantes universitarias, UNEMI 2018: resultados del plan piloto}

Vol. 3, núm. 1., (2019)

Mariela Libelly Lozada Meza; Lorena Paola Ramírez Morán; Edwin Hernán Alvarado Chicaíza; Carol Johana Cajas Bejarano

nacer, retardo del crecimiento intrauterino (RCIU) y recién nacido pequeño para edad gestacional (RNPEG). La evaluación de seguimiento tiene como finalidades observar el cumplimiento de las indicaciones realizadas en la evaluación inicial, conocer los aspectos que dificultaron dicho cumplimiento y realizar los correctivos necesarios de manera precoz, de tal manera que se logré un manejo nutricional adecuado durante este periodo.

Hernández Días (2016), fundamenta que el estado nutricional de la gestante al momento de la captación, su composición corporal grasa y las ganancias de peso durante el embarazo, pueden incidir en el crecimiento y desarrollo fetal y en la salud materna. Cuando se pregunta cuánto peso debería ganar la gestante su trabajo concluye que: la causa del incremento de peso es que durante la gestación se produce la síntesis de nuevos tejidos: glándulas mamarias, engrosamiento del tejido uterino, aumento del tejido adiposo y desarrollo del feto y de la placenta. También es necesaria la acumulación de masa grasa para sustentar el crecimiento del bebé durante el embarazo y la lactancia, que, en general, se localiza en el pecho, los muslos y el abdomen. Sin embargo, aunque el aumento de peso es necesario, este debe ser adecuado y controlado.

Es aconsejable que la mujer tenga un peso adecuado antes del embarazo. Si es así, durante los 9 meses de gestación se debe aumentar de media entre 9 y $12 \mathrm{Kg}$, pero depende mucho de cada situación y de cada persona. En el primer trimestre, la subida de peso medio es entre 1-3 kg, por el aumento de reservas. Aunque hay mujeres que incluso adelgazan debido a las náuseas y los vómitos que aparecen. Durante el segundo y tercer trimestre es cuando se produce la mayor subida de peso, por el crecimiento mayor del feto y los tejidos, así como los depósitos de grasa 


\section{Evaluación del estado nutricional de gestantes universitarias, UNEMI 2018:}

resultados del plan piloto

Vol. 3, núm. 1., (2019)

Mariela Libelly Lozada Meza; Lorena Paola Ramírez Morán; Edwin Hernán Alvarado Chicaíza;

Carol Johana Cajas Bejarano

de cara a la lactancia materna. El Índice de masa corporal (IMC) es una fórmula muy sencilla para determinar el grado de peso de la mujer en función de su estatura, pero no es determinante porque no nos dice el porcentaje de grasa.

La valoración antropométrica es la medición del peso en kilogramos (Peso- Kg.); y talla en metros al cuadrado (Talla- m2). el cálculo del Índice de Masa Corporal (IMC) aplica la fórmula:

$$
I M C=\frac{\text { Peso }(\mathrm{kg})}{\text { Talla }\left(\mathrm{m}^{2}\right)}
$$

\section{Clasificación del IMC según estado nutricional de la gestante}

\begin{tabular}{ll}
\hline IMC & Categoría \\
\hline IMC $<20 \mathrm{~kg} / \mathrm{m}^{2}$ & Enflaqueci \\
IMC $20-24.9$ & Normal \\
IMC $25-29 \mathrm{~kg} / \mathrm{m}^{2}$ & Sobrepeso \\
IMC $>30 \mathrm{~kg} / \mathrm{m}^{2}$ & Obesidad \\
\hline
\end{tabular}

Fuente: Recuperado de (Minjarez, et al 2013). Ganancia de peso gestacional como factor de riesgo para desarrollar complicaciones obstétricas.

El diagnóstico de estado nutricional con la aplicación de métodos antropométrico considera algunos términos:

- Peso preconcepcional: "Influye en el estado nutricional de la embarazada, en forma independiente al incremento de peso materno; este es el mejor predictor de peso del niño al nacer y relaciona con el peso fetal.”. Se obtiene en el primer trimestre; este valor tiene importancia como parámetro per se para la toma de decisiones. 


\section{Evaluación del estado nutricional de gestantes universitarias, UNEMI 2018: resultados del plan piloto}

Vol. 3, núm. 1., (2019)

Mariela Libelly Lozada Meza; Lorena Paola Ramírez Morán; Edwin Hernán Alvarado Chicaíza; Carol Johana Cajas Bejarano

- Talla Materna: Fescina et al. (2011) es un predictor de riesgo de retardo de crecimiento uterino, cuando mida de 140 a $150 \mathrm{~cm}$. El pronóstico es diferente si depende de una baja talla genética. Cuando la talla baja es de origen familiar tiene el riesgo de complicarse en el parto por una des proposición cefalopelvica. Los estudios realizados indican que los niños recién nacidos de bajo peso para la edad clasifican como adecuados cuando se ajustan por talla materna.

- Incremento de peso materno: Para Worthington (2012) "Aumento de peso materno es un promedio total que aumenta una mujer durante este periodo es de aproximadamente $12.5 \%$ con un promedio de $0.5 \mathrm{~kg}$. por semana." El crecimiento fetal no depende de los nutrientes de la dieta, sino al transportar los nutrientes a través de la placenta igualmente importante.

\section{Distribución de la ganancia de peso en el embarazo}

\begin{tabular}{lll}
\hline Feto & $24 \%$ & $3000 \mathrm{~g}$ \\
Placenta & $5 \%$ & $600 \mathrm{~g}$ \\
Líquido amniótico & $6 \%$ & $800 \mathrm{~g}$ \\
Útero, mamas y sangre & $20 \%$ & $2500 \mathrm{~g}$ \\
Agua extracelular & $16 \%$ & $2000 \mathrm{~g}$ \\
Depósito de grasa & $29 \%$ & $3600 \mathrm{~g}$ \\
\hline
\end{tabular}

Fuente: Recuperado de Dirección Nacional de Nutrición PANN. (MSP, 2000)

El indicador bioquímico considerado para establecer estado nutricional en el presente estudio fue copiar información del valor de hemoglobina registrado en el formulario 51A - 2011 del Ministerio de Salud Pública (MSP, 2016), independiente si eran monitoreadas en el servicio 


\section{Evaluación del estado nutricional de gestantes universitarias, UNEMI 2018:}

resultados del plan piloto

Vol. 3, núm. 1., (2019)

Mariela Libelly Lozada Meza; Lorena Paola Ramírez Morán; Edwin Hernán Alvarado Chicaíza;

Carol Johana Cajas Bejarano

publico o privado, respecto a esta información la revisión de los expertos hizo que este literal fuese considerado solo como un recordatorio verbal de la gestante por que dicho formulario permace en los centros de atención médica, considerando entonces que el cuestionamiento fuera si la estudiante fue indicada en su último control si presentava anemia, no presentava o no lo sabia, y de haber sido informada como catalogó el profesional si una anemia leve moderada o severa, procediendo en la tabulacion de resultados a la clasificación con los rangos establecidos por el Ministerio de Salud en el Ecuador.

Técnica de análisis de datos

El análisis de los datos se realizó con ayuda de los paquetes estadísticos EXCEL® y el programa Statistical Package for the Social Sciences (SPSS®) versión 21. Además, se realizaron las respectivas pruebas de adecuación muestral de los datos disponibles y las pruebas para determinar la confiabilidad y validez de la encuesta para el caso en estudio.

\section{Resultados.}

A. Datos informativos de las estudiantes gestantes.

En el siguiente analisis estadístico se identificó algunos factores que pueden incidir en el estado nutricional de la gestante: según el lugar de procedencia con codificación de 1. Milagro, 2. Otro cantón, 3. Otra provincia, se obtuvo como resultado que el $65 \%$ procedían de otro cantón y el $35 \%$ de Milagro. 


\section{Evaluación del estado nutricional de gestantes universitarias, UNEMI 2018: resultados del plan piloto}

Vol. 3, núm. 1., (2019)

Mariela Libelly Lozada Meza; Lorena Paola Ramírez Morán; Edwin Hernán Alvarado Chicaíza; Carol Johana Cajas Bejarano

Según el estado civil con codificación de 1. Soltera, 2. Casada, 3. Unión libre, 4. Divorciada, 5. Viuda, se obtuvo como resultado que el $60 \%$ estaban en unión libre, el $30 \%$ casadas, y el $10 \%$ solteras.

Según el soporte económico con codificación de 1. Padres, 2. Autofinanciamiento, 3. Pareja, 4. Otros familiares, 5. De todos, se obtuvo que el $90 \%$ dependían de la pareja, mientras que el $10 \%$ autofinanciamiento.

Según la convivencia actual con codificación de 1. Padres, 2. Compañeros, 3. Esposo o pareja, 4. Otros familiares, 5. Sola, se obtuvo que el 90\% vivía con el esposo y el 10\% sola.

Según el rango de edad con codificación de 1. De 15 a 19 años, 2. De 20 a 24 años, 3. De 25 a 29 años, 4. De 30 a 34 años, 5 . $\geq 35$ años, se obtuvo como resultado que el $50 \%$ estaba en un rango de 20 a 24 años, el $25 \%$ en un rango de 25 a 29 años, el $20 \%$ en un rango de 15 a 19 años y el 5\% en un rango de 30 a 34 años.

Tabla 1. Aspectos informativos de las Estudiantes Gestantes

\begin{tabular}{cccccc}
\hline CÓDIGO & $\begin{array}{c}\text { LUGAR DE } \\
\text { PROCEDENC }\end{array}$ & $\begin{array}{c}\text { ESTAD } \\
\text { O } \\
\text { CIVIL }\end{array}$ & $\begin{array}{c}\text { SOPORTE } \\
\text { ECONÓMIC } \\
\text { IA }\end{array}$ & $\begin{array}{c}\text { CONVIVEN } \\
\text { CIA } \\
\text { ACTUAL }\end{array}$ & $\begin{array}{c}\text { RANGO } \\
\text { DE } \\
\text { EDAD }\end{array}$ \\
\hline EENEGU-001 & 2 & 3 & 3 & 3 & 2 \\
EENEGU-002 & 2 & 3 & 3 & 3 & 2 \\
EENEGU-003 & 2 & 3 & 3 & 3 & 2 \\
EENEGU-004 & 2 & 3 & 3 & 3 & 3 \\
EENEGU-005 & 2 & 3 & 3 & 3 & 2 \\
EENEGU-006 & 1 & 2 & 3 & 3 & 3 \\
EENEGU-007 & 2 & 3 & 3 & 3 & 1 \\
EENEGU-008 & 2 & 2 & 3 & 3 & 1 \\
EENEGU-009 & 1 & 2 & 3 & 3 & 1 \\
EENEGU-010 & 2 & 2 & 3 & 3 & 2 \\
EENEGU-011 & 2 & 3 & 3 & 3 & 2
\end{tabular}


Evaluación del estado nutricional de gestantes universitarias, UNEMI 2018: resultados del plan piloto

Vol. 3, núm. 1., (2019) Mariela Libelly Lozada Meza; Lorena Paola Ramírez Morán; Edwin Hernán Alvarado Chicaíza;

Carol Johana Cajas Bejarano

\begin{tabular}{llllll} 
EENEGU-012 & 1 & 3 & 3 & 3 & 3 \\
EENEGU-013 & 2 & 3 & 3 & 3 & 2 \\
EENEGU-014 & 2 & 1 & 2 & 5 & 4 \\
EENEGU-015 & 1 & 2 & 3 & 3 & 1 \\
EENEGU-016 & 1 & 2 & 3 & 3 & 3 \\
EENEGU-017 & 1 & 3 & 3 & 3 & 2 \\
EENEGU-018 & 2 & 1 & 2 & 5 & 2 \\
EENEGU-019 & 2 & 3 & 3 & 3 & 3 \\
EENEGU-020 & 1 & 3 & 3 & 3 & 2 \\
\hline
\end{tabular}

Fuente: Muestra de prueba piloto y validación de instrumentos de estado nutricional en estudiantes gestantes universitarias, UNEMI 2018.

Figura 1. Aspectos informativos de las gestantes

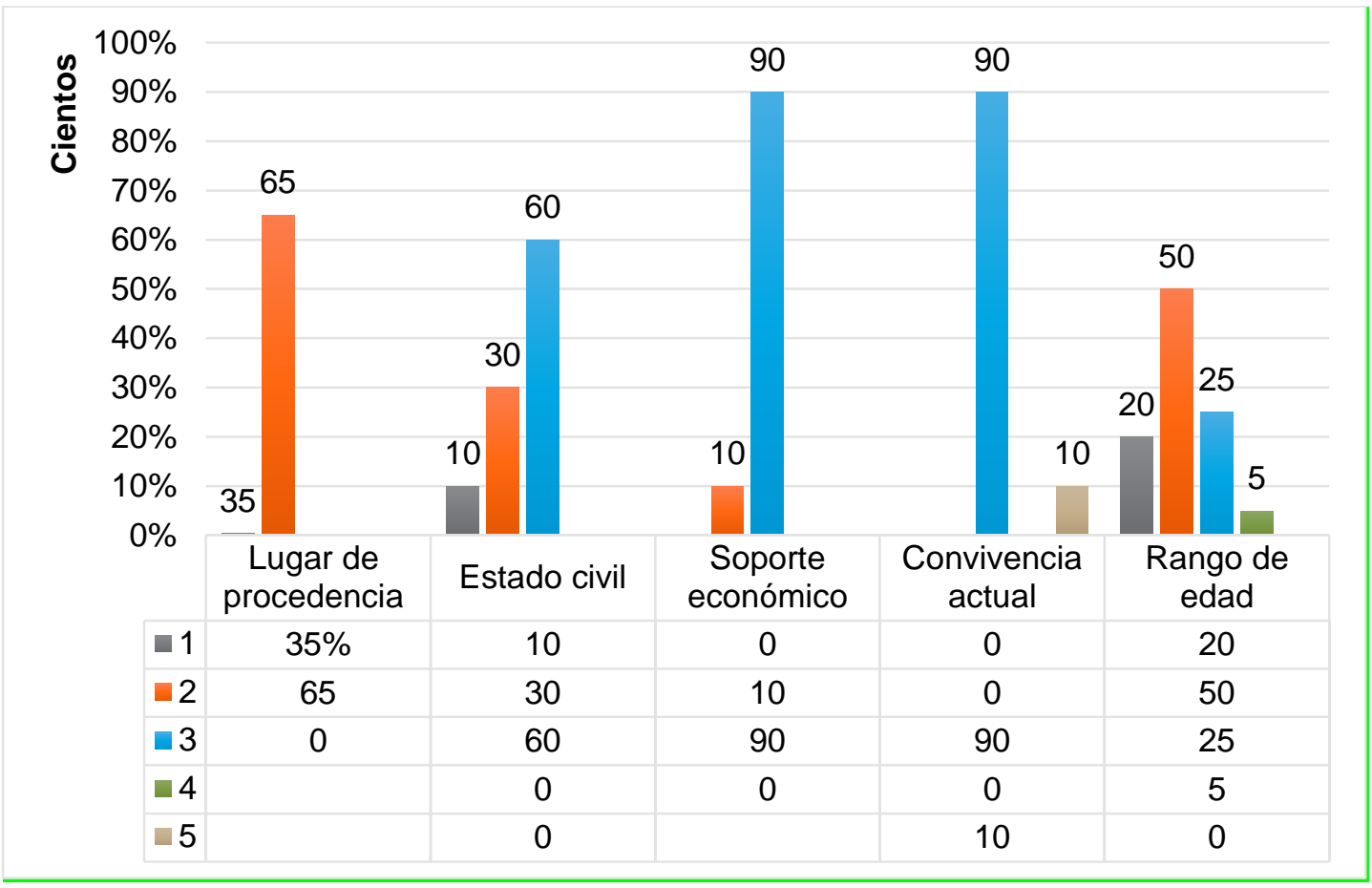

Fuente: Muestra de prueba piloto y validación de instrumentos de estado nutricional en estudiantes gestantes universitarias, UNEMI 2018. 
Evaluación del estado nutricional de gestantes universitarias, UNEMI 2018: resultados del plan piloto

Vol. 3, núm. 1., (2019)

Mariela Libelly Lozada Meza; Lorena Paola Ramírez Morán; Edwin Hernán Alvarado Chicaíza; Carol Johana Cajas Bejarano

\section{B. Estado nutricional - método con valoraciónantropométrica}

Los resultados obtenidos sobre el Índice de Masa Corporal (IMC) según edad gestacional en estudiantes gestantes indican que del 100\%, el $45 \%$ presenta sobrepeso, el $40 \%$ presenta peso normal o adecuado, el $10 \%$ presenta obesidad y el $5 \%$ presenta bajo peso para la edad gestacional.

Tabla 2. Valoración antropométrica

\begin{tabular}{|c|c|c|c|c|c|}
\hline CÓDIGO & $\begin{array}{c}\text { SEMANAS } \\
\text { DE } \\
\text { GESTACI } \\
\text { ÓN }\end{array}$ & $\begin{array}{c}\text { PESO } \\
\text { ACTUAL }\end{array}$ & TALLA & $\begin{array}{c}\text { IMC/EDAD } \\
\text { GESTACION } \\
\text { AL }\end{array}$ & INTERPRETACIÓN \\
\hline EENEGU-001 & 24 & 72,5 & 1,57 & 29,41 & S \\
\hline EENEGU-002 & 18 & 65,5 & 1,61 & 25,27 & S \\
\hline EENEGU-003 & 20 & 72,7 & 1,64 & 27,03 & S \\
\hline EENEGU-004 & 36 & 62,6 & 1,60 & 24,45 & $\mathrm{~N}$ \\
\hline EENEGU-005 & 24 & 64,2 & 1,53 & 27,43 & S \\
\hline EENEGU-006 & 18 & 69,4 & 1,45 & 33,01 & $\mathrm{O}$ \\
\hline EENEGU-007 & 26 & 70 & 1,64 & 26,03 & S \\
\hline EENEGU-008 & 37 & 66,4 & 1,62 & 25,30 & S \\
\hline EENEGU-009 & 11 & 49,5 & 1,58 & 19,83 & $\mathrm{BP}$ \\
\hline EENEGU-010 & 30 & 64,5 & 1,47 & 29,85 & S \\
\hline EENEGU-011 & 37 & 69,5 & 1,60 & 27,15 & S \\
\hline EENEGU-012 & 14 & 55,4 & 1,62 & 21,11 & $\mathrm{~N}$ \\
\hline EENEGU-013 & 18 & 51,2 & 1,50 & 22,76 & $\mathrm{~N}$ \\
\hline EENEGU-014 & 12 & 63,5 & 1,64 & 23,61 & $\mathrm{~N}$ \\
\hline EENEGU-015 & 16 & 56,6 & 1,56 & 23,26 & $\mathrm{~N}$ \\
\hline EENEGU-016 & 26 & 55,3 & 1,53 & 23,62 & $\mathrm{~N}$ \\
\hline EENEGU-017 & 38 & 58,5 & 1,58 & 23,43 & $\mathrm{~N}$ \\
\hline EENEGU-018 & 20 & 75,3 & 1,57 & 30,55 & $\mathrm{O}$ \\
\hline
\end{tabular}


Evaluación del estado nutricional de gestantes universitarias, UNEMI 2018: resultados del plan piloto

Vol. 3, núm. 1., (2019) Mariela Libelly Lozada Meza; Lorena Paola Ramírez Morán; Edwin Hernán Alvarado Chicaíza; Carol Johana Cajas Bejarano

\begin{tabular}{llllll}
\hline EENEGU-019 & 18 & 61 & 1,50 & 27,11 & $\mathrm{~S}$ \\
EENEGU-020 & 24 & 59 & 1,59 & 23,34 & $\mathrm{~N}$ \\
\hline
\end{tabular}

Fuente: Muestra de prueba piloto y validación de instrumentos de estado nutricional en estudiantes gestantes universitarias, UNEMI 2018.

\section{Figura 2. Valoración antropométrica}

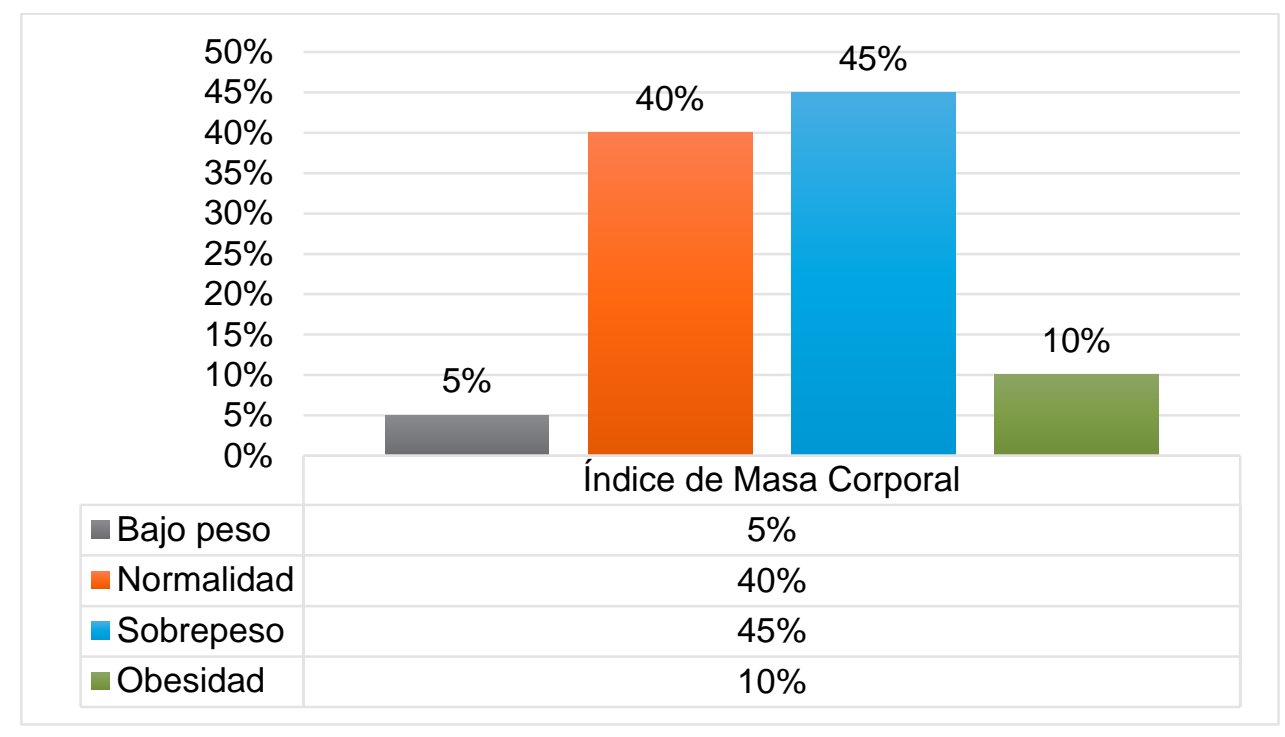

Fuente: Muestra de prueba piloto y validación de instrumentos de estado nutricional en estudiantes gestantes universitarias, UNEMI 2018

Tabla 3 Correlación de variables

\begin{tabular}{|c|c|c|c|c|c|}
\hline & & & $\begin{array}{l}\text { Interpretación } \\
\text { gestacional }\end{array}$ & IMC/Edad & Edad \\
\hline \multirow{2}{*}{$\begin{array}{l}\text { Interpretación } \\
\text { gestacional }\end{array}$} & \multirow[t]{2}{*}{ IMC/Edad } & $\begin{array}{l}\begin{array}{l}\text { Coeficiente } \\
\text { correlación }\end{array} \\
\end{array}$ & 1,000 & &,$- 341 * *$ \\
\hline & & Sig. (bilateral) & & & ,004 \\
\hline Edad & & $\begin{array}{l}\text { Coeficiente } \\
\text { correlación }\end{array} \quad$ de &,$- 341 * *$ & & 1,000 \\
\hline
\end{tabular}




\section{Evaluación del estado nutricional de gestantes universitarias, UNEMI 2018: resultados del plan piloto}

Vol. 3, núm. 1., (2019)

Mariela Libelly Lozada Meza; Lorena Paola Ramírez Morán; Edwin Hernán Alvarado Chicaíza; Carol Johana Cajas Bejarano

Sig. (bilateral) $\quad, 004$

Al comparar edad con la interpretación del IMC se encuentra una correlación negativa media.

Los resultados obtenidos en la encuesta sobre si conocían las estudiantes gestante del padecimiento de anemia según el diagnóstico del control médico que hubieren tenido previo al presente estudio cuyo diagnóstico en el servicio público es registrado en el formulario 51A 2011 del MSP y en centros particulares en e carnet de salud de la gestante; del total de participantes el 35\% recuerdan haber sido indicada que presenta anemia, en tanto que un $45 \%$ no refiere tener anemia, mientras que el $20 \%$ desconocían si presentaban anemia, como se detalla en la figura 3. Cabe mencionar que de las estudiantes que, si presentaban anemia, no todas recordaban el valor de hemoglobina

Figura 3. Padecimiento de Anemia

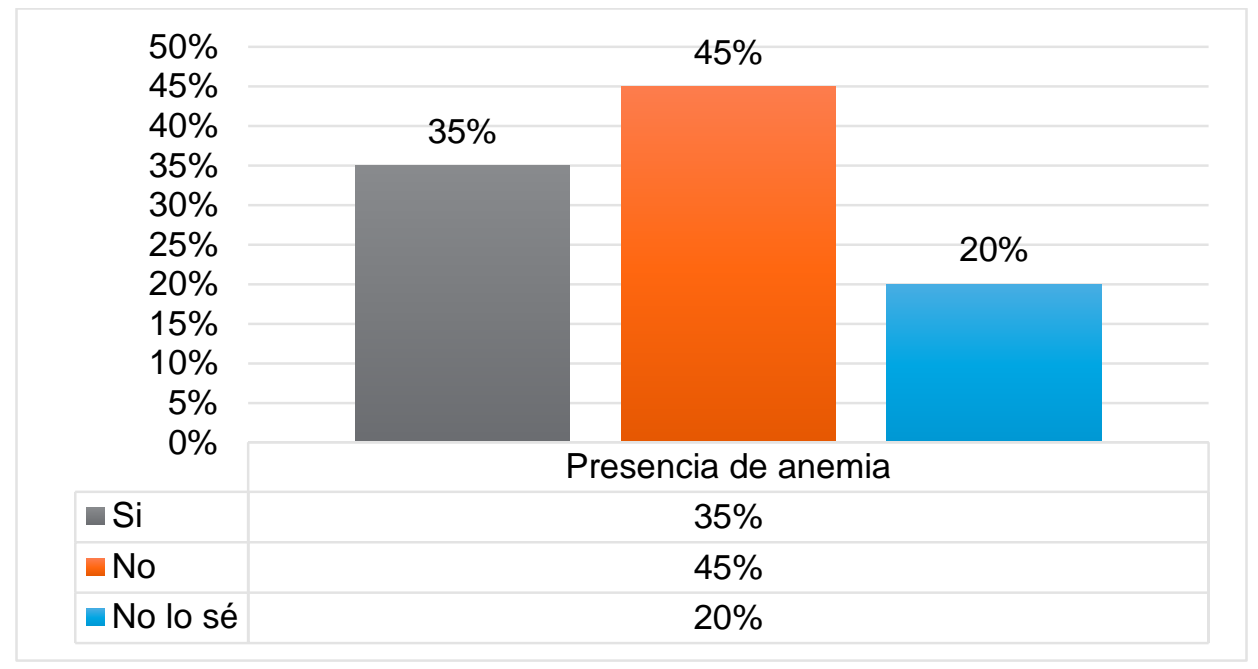

Fuente: Muestra de prueba piloto y validación de instrumentos de estado nutricional en estudiantes gestantes universitarias, UNEMI 2018 


\section{Evaluación del estado nutricional de gestantes universitarias, UNEMI 2018:}

resultados del plan piloto

Vol. 3, núm. 1., (2019)

Mariela Libelly Lozada Meza; Lorena Paola Ramírez Morán; Edwin Hernán Alvarado Chicaíza;

Carol Johana Cajas Bejarano

Según los datos aportados por las estudiantes gestantes que indicaron conocer que presentabam anemia los valores son los que se detallan en la tabla 3 , se consideraría que de no contar con los dados de todas las participantes se presentaría un sesgo en el estudio refiriendo retirar el criterio de valoración de estado nutricional con el método bioquímico.

Con lo referido se establece o no la presencia de anemia según trimestre de gestación, con la corrección según altitud (metros sobre el nivel del mar), como se muestra en la figura 4.

Figura 4. Padecimiento de Anemia por trimestre de embarazo

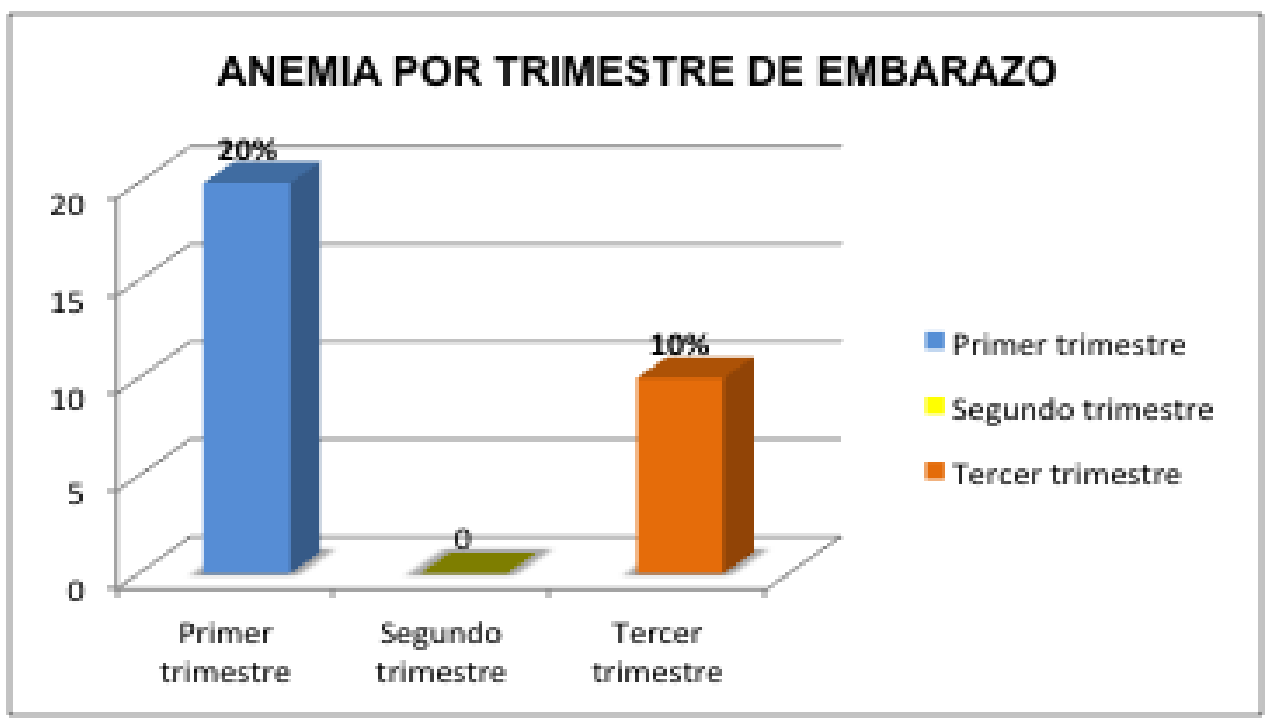

Fuente: Muestra de prueba piloto y validación de instrumentos de estado nutricional en estudiantes gestantes universitarias, UNEMI 2018

Tabla 4. Correlación de variables

\begin{tabular}{llll}
$\begin{array}{l}\text { Presencia } \\
\text { anemia }\end{array}$ & de & $\begin{array}{l}\text { Semana } \\
\text { gestación }\end{array}$ & de \\
\hline
\end{tabular}




\section{Evaluación del estado nutricional de gestantes universitarias, UNEMI 2018: resultados del plan piloto}

Vol. 3, núm. 1., (2019)

Mariela Libelly Lozada Meza; Lorena Paola Ramírez Morán; Edwin Hernán Alvarado Chicaíza; Carol Johana Cajas Bejarano

\begin{tabular}{|c|c|c|c|c|}
\hline \multirow{2}{*}{$\begin{array}{l}\text { Presencia } \\
\text { anemia }\end{array}$} & \multirow[t]{2}{*}{ de } & $\begin{array}{l}\text { Coeficiente } \\
\text { correlación }\end{array}$ & 1,000 &,- 137 \\
\hline & & Sig. (bilateral) & & ,223 \\
\hline \multirow{2}{*}{$\begin{array}{l}\text { Semana } \\
\text { gestación }\end{array}$} & \multirow[t]{2}{*}{ de } & $\begin{array}{l}\text { Coeficiente } \\
\text { correlación }\end{array}$ &,- 137 & 1,000 \\
\hline & & Sig. (bilateral) &, 223 & \\
\hline
\end{tabular}

Fuente: Muestra de prueba piloto y validación de instrumentos de estado nutricional en estudiantes gestantes universitarias, UNEMI 2018

Entre las variables presencia de anemia y semana de gestación se encuentra una correlación negativa media. Al colocarle el valor 1 a la respuesta 'Si' en presencia de anemia, el resultado indica que mientras avanzan las semanas de gestación, disminuye el valor de presencia de anemia que corresponde al 'Si'.

\section{Método dietético}

Al determinar estado nutricional en gestantes se ha considerado algunos aspectos que identifican patrón de consumo alimentario con el análisis de preguntas como:¡usted desayuna? con codificación de 1 . Siempre, 2. A veces, 3. Nunca, se obtuvo como resultado que el $65 \%$ siempre desayunaba, mientras que el $35 \%$ a veces lo hacía.

Según los tiempos de comida que realizaban al día con codificación de $1 . \leq 3,2.4$ a 5, 3 . $\geq 6$, se obtuvo como resultado que el $65 \%$ comía de 4 a 5 veces al día, mientras que el $35 \%$ solo hacia $\leq 3$ veces al día. 
Evaluación del estado nutricional de gestantes universitarias, UNEMI 2018:

resultados del plan piloto

Vol. 3, núm. 1., (2019)

Mariela Libelly Lozada Meza; Lorena Paola Ramírez Morán; Edwin Hernán Alvarado Chicaíza;

Carol Johana Cajas Bejarano

Según la pregunta ¿considera que su dieta es adecuada para la gestación? Con codificación 1. Si, 2. No, 3. No lo sé, se obtuvo como resultado que el $65 \%$ no lo sabía, el $20 \%$ consideraba que no era adecuada y el 15\% estimaba que si era adecuada para la gestación.

Según la autopercepción de las características que tenía su dieta con codificación de 1 . Variada, 2. Completa, 3. Suficiente, 4. Inocua, 5. Selectiva, se obtuvo como resultado que el 75\% era variada, el $15 \%$ era suficiente y el 10\% era completa.

Según el resultado del porcentaje de adecuación con respecto a la dieta consumida en 24 horas con codificación de 1. Adecuada, 2. Deficiente, 3. Excesiva, se obtuvo como resultado que el $60 \%$ era deficiente, mientras que el $20 \%$ era adecuada. Lo referido se describe criterios en la tabla 5 y los resultados en la figura 4.

\section{Tabla 5. Valoración alimentaria}

\begin{tabular}{|c|c|c|c|c|c|}
\hline & USTED & TIEMPO & CONSIDERA & CARACTERIS & VALORACIÓ \\
\hline CÓDIGO & $\begin{array}{l}\text { DESAYU } \\
\text { NA }\end{array}$ & $\begin{array}{l}\text { S DE } \\
\text { COMIDA }\end{array}$ & $\begin{array}{l}\text { QUE SU DIETA } \\
\text { ES ADECUADA }\end{array}$ & $\begin{array}{l}\text { TICAS DE LA } \\
\text { DIETA }\end{array}$ & $\begin{array}{l}\mathbf{N} \text { DE } \\
\text { DIETA }\end{array}$ \\
\hline $\begin{array}{l}\text { EENEGU- } \\
001\end{array}$ & 10 & chinnon & 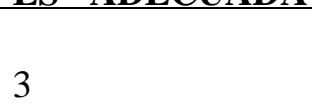 & Dinis & Donar \\
\hline $\begin{array}{l}\text { EENEGU- } \\
002\end{array}$ & 1 & 2 & 3 & 1 & D \\
\hline $\begin{array}{l}\text { EENEGU- } \\
003\end{array}$ & 1 & 2 & 3 & 1 & D \\
\hline $\begin{array}{l}\text { EENEGU- } \\
004\end{array}$ & 1 & 1 & 1 & 1 & D \\
\hline $\begin{array}{l}\text { EENEGU- } \\
005\end{array}$ & 1 & 1 & 3 & 1 & D \\
\hline $\begin{array}{l}\text { EENEGU- } \\
006\end{array}$ & 1 & 2 & 2 & 1 & D \\
\hline $\begin{array}{l}\text { EENEGU- } \\
007\end{array}$ & 2 & 2 & 3 & 1 & A \\
\hline
\end{tabular}


Evaluación del estado nutricional de gestantes universitarias, UNEMI 2018: resultados del plan piloto

Vol. 3, núm. 1., (2019)

Mariela Libelly Lozada Meza; Lorena Paola Ramírez Morán; Edwin Hernán Alvarado Chicaíza; Carol Johana Cajas Bejarano

\begin{tabular}{|c|c|c|c|c|c|}
\hline $\begin{array}{l}\text { EENEGU- } \\
008\end{array}$ & 2 & 2 & 3 & 1 & $\mathrm{D}$ \\
\hline $\begin{array}{l}\text { EENEGU- } \\
009\end{array}$ & 2 & 1 & 2 & 3 & A \\
\hline $\begin{array}{l}\text { EENEGU- } \\
010\end{array}$ & 2 & 1 & 1 & 1 & $\mathrm{D}$ \\
\hline $\begin{array}{l}\text { EENEGU- } \\
011\end{array}$ & 1 & 2 & 3 & 3 & D \\
\hline $\begin{array}{l}\text { EENEGU- } \\
012\end{array}$ & 1 & 2 & 2 & 2 & A \\
\hline $\begin{array}{l}\text { EENEGU- } \\
013\end{array}$ & 1 & 1 & 3 & 1 & $\mathrm{D}$ \\
\hline $\begin{array}{l}\text { EENEGU- } \\
014\end{array}$ & 2 & 1 & 3 & 1 & $\mathrm{D}$ \\
\hline $\begin{array}{l}\text { EENEGU- } \\
015\end{array}$ & 1 & 2 & 2 & 1 & $\mathrm{D}$ \\
\hline $\begin{array}{l}\text { EENEGU- } \\
016\end{array}$ & 2 & 2 & 3 & 1 & D \\
\hline $\begin{array}{l}\text { EENEGU- } \\
017\end{array}$ & 1 & 2 & 3 & 2 & D \\
\hline $\begin{array}{l}\text { EENEGU- } \\
018\end{array}$ & 1 & 2 & 3 & 1 & A \\
\hline $\begin{array}{l}\text { EENEGU- } \\
019\end{array}$ & 1 & 2 & 1 & 1 & $\mathrm{D}$ \\
\hline $\begin{array}{l}\text { EENEGU- } \\
020\end{array}$ & 1 & 2 & 3 & 3 & $\mathrm{D}$ \\
\hline
\end{tabular}

Fuente: Muestra de prueba piloto y validación de instrumentos de estado nutricional en estudiantes gestantes universitarias, UNEMI 2018. 


\section{Evaluación del estado nutricional de gestantes universitarias, UNEMI 2018:}

resultados del plan piloto

Vol. 3, núm. 1., (2019)

Mariela Libelly Lozada Meza; Lorena Paola Ramírez Morán; Edwin Hernán Alvarado Chicaíza;

Carol Johana Cajas Bejarano

\section{Figura 4. Actitud y práctica alimentaria}
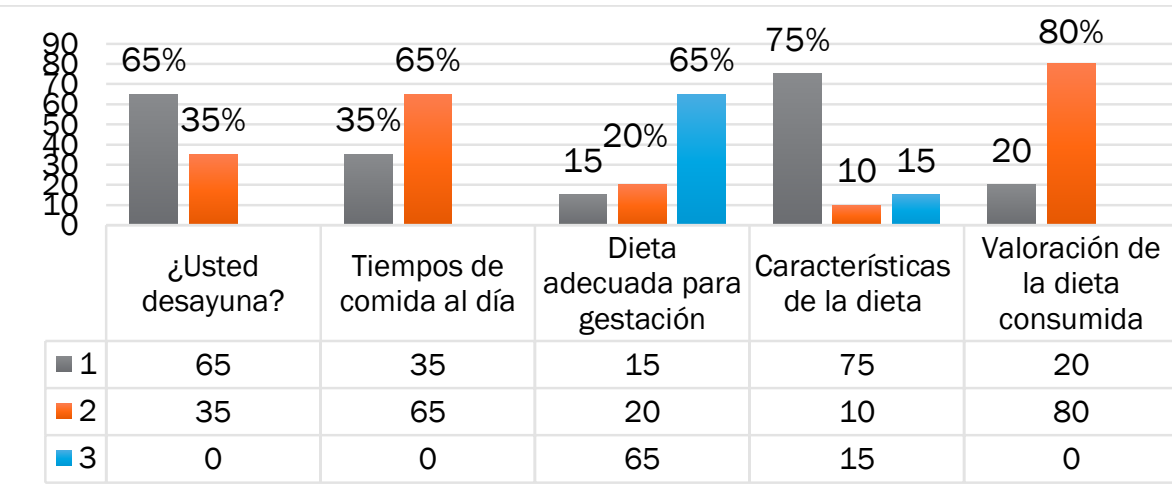

Análisis de aporte nutrimental de calorías, macronutrientes y hierro. Los resultados obtenidos según la valoración de la dieta consumida en 24 horas indican que del 100\% de las gestantes, el $80 \%$ de la dieta es deficiente, mientras que el $20 \%$ es adecuada. Con respecto al consumo excesivo en la dieta no se hallaron resultados en relación a esta dimensión.

Tabla 6 Evaluación de aporte nutrimental en porcentaje de adecuación

\begin{tabular}{|c|c|c|c|c|c|}
\hline VALORACION DE & \multicolumn{3}{|c|}{ NÚMERO } & & \\
\hline Adecuada & \multicolumn{2}{|c|}{4} & & \multicolumn{2}{|l|}{$20 \%$} \\
\hline Deficiente & \multicolumn{2}{|c|}{16} & & \multicolumn{2}{|l|}{$80 \%$} \\
\hline Excesiva & \multicolumn{2}{|c|}{0} & & \multicolumn{2}{|l|}{$0 \%$} \\
\hline Total & \multicolumn{2}{|c|}{20} & & \multicolumn{2}{|c|}{$100 \%$} \\
\hline Media & Mediana & $\begin{array}{l}\text { Desviación } \\
\text { estándar }\end{array}$ & Rango & Mínimo & Máximo \\
\hline 2094,05 & 2114,00 & 523,985 & 2765 & 660 & 3425 \\
\hline
\end{tabular}




\section{Evaluación del estado nutricional de gestantes universitarias, UNEMI 2018: resultados del plan piloto}

Vol. 3, núm. 1., (2019)

Mariela Libelly Lozada Meza; Lorena Paola Ramírez Morán; Edwin Hernán Alvarado Chicaíza; Carol Johana Cajas Bejarano

$\begin{array}{lcccccc}\text { Proteína } & 318,21 & 327,00 & 117,197 & 717 & 56 & 773 \\ \text { Grasas } & 569,47 & 569,00 & 212,897 & 923 & 116 & 1039 \\ \begin{array}{l}\text { Hidratos de } \\ \text { carbono }\end{array} & 1198,00 & 1222,00 & 306,412 & 1537 & 425 & 1962 \\ \text { Hierro } & 19,6077 & 18,0000 & 11,00027 & 82,00 & 4,00 & 86,00\end{array}$

Fuente: Muestra de prueba piloto y validación de instrumentos de estado nutricional en estudiantes gestantes universitarias, UNEMI 2018

Frecuencia de consumo de alimentos en la semana, registra 15 grupos alimenticios que contienen los alimentos de mayor consumo en Latinoamérica y particular para la región de donde provienen las gestantes universitarias grupo objetivo de estudio. Los resultados obtenidos del cuestionario de frecuencia por grupos de alimentos, indican el siguiente consumo promedio de alimentos en la semana:

- Grupo 1 (Leche y derivados): consumo promedio 3 veces por semana.

- Grupo 2 (Huevo): consumo promedio 3 veces por semana.

- Grupo 3 (Carnes y vísceras): consumo promedio 4 veces por semana.

- Grupo 4 (Embutidos): consumo promedio 2 veces por semana

- Grupo 5 (Pescados y mariscos): consumo promedio 2 veces por semana.

- Grupo 6 (Leguminosas y oleaginosas): consumo promedio 4 veces por semana. 
Evaluación del estado nutricional de gestantes universitarias, UNEMI 2018: resultados del plan piloto

Vol. 3, núm. 1., (2019)

Mariela Libelly Lozada Meza; Lorena Paola Ramírez Morán; Edwin Hernán Alvarado Chicaíza;

Carol Johana Cajas Bejarano

- Grupo 7 (Semillas): consumo promedio 1 vez por semana.

- Grupo 8 (Especias): consumo promedio 2 veces por semana.

- Grupo 9 (Verduras, hortalizas y otros vegetales): consumo promedio 5 veces por semana.

- Grupo 10 (Frutas): consumo promedio 4 veces por semana.

- Grupo 11 (Tubérculos y raíces): consumo promedio 4 veces por semana

- Grupo 12 (Cereales, harinas, pastas y otros derivados): consumo promedio 7 veces por semana.

- Grupo 13 (Azucares): consumo promedio 6 veces por semana.

- Grupo 14 (Grasas): consumo promedio 4 veces por semana.

- Grupo 15 (Otros productos): consumo promedio 5 veces por semana.

Tabla 7 Frecuencia de consumo de alimentos

\begin{tabular}{llllll}
\hline & Media & $\begin{array}{l}\text { Desviación } \\
\text { estándar }\end{array}$ & Rango & Mínimo & Máximo \\
\hline Grupo 1 & 4,14 & 2,09 & 9 & 1 & 10 \\
Grupo 2 & 3,02 & 1,91 & 9 & 0 & 9 \\
Grupo 3 & 5,27 & 2,564 & 11 & 0 & 11 \\
Grupo 4 & 1,69 & 1,158 & 5 & 0 & 5 \\
Grupo 5 & 2,67 & 1,75 & 9 & 0 & 8 \\
Grupo 6 & 3,53 & 1,865 & 8 & 0 & 8 \\
Grupo 7 & 1,23 & 1,770 & 7 & 0 & 7 \\
Grupo 8 & 2,43 & 2,56 & 9 & 0 & 9 \\
Grupo 9 & 4,54 & 2,28 & 10 & 0 & 10 \\
Grupo 10 & 5,88 & 3,25 & 15 & 0 & 15
\end{tabular}




\section{Evaluación del estado nutricional de gestantes universitarias, UNEMI 2018: resultados del plan piloto}

Vol. 3, núm. 1., (2019)

Mariela Libelly Lozada Meza; Lorena Paola Ramírez Morán; Edwin Hernán Alvarado Chicaíza; Carol Johana Cajas Bejarano

\begin{tabular}{llllll} 
Grupo 11 & 3,21 & 2,34 & 12 & 0 & 12 \\
Grupo 12 & 9,42 & 4,35 & 21 & 1 & 22 \\
Grupo 13 & 4,62 & 2,62 & 10 & 0 & 10 \\
Grupo 14 & 3,74 & 2,40 & 7 & 0 & 7 \\
Grupo 15 & 4,38 & 2,97 & 13 & 0 & 13 \\
\hline
\end{tabular}

Fuente: Muestra de prueba piloto y validación de instrumentos de estado nutricional en estudiantes gestantes universitarias, UNEMI 2018

\section{Conclusiones.}

En conclusión se determinó que entre los datos generales de las estudiantes gestantes que pueden incidir en su estado nutricional es que, en su mayorìa provenían de cantoles distantes a la Institución aducativa donde permanecen la jornada escolar, además dependen economicamente de su pareja $\mathrm{y}$, un porcentaje significativo aun eran adolescentes y adultas jóvenes.

Se identificó que el patrón alimentario evaluado por las actitudes y prácticas de consumo habitual y frecuencia de alimentos en la dieta llevo a la gestante a presentar en más de la mitad algún grado de malnutrición sea al sobrepeso u obesidad o bajo péso.

Por otro lado, se consideró que una complicación recurrente en la gestante es la anemia ferropénica, sin embargo, debido a la no disponibilidad de los valores de henoglobina en todas las participantes por desconocimiento, o por no contar con la prueba respectiva el resultado se ve sesgado

Por lo tanto, se concluye que la prueba piloto ha empleado instrumentos que han obtenido índices de validación comparables a otras herramientas de aplicación en la 


\section{Evaluación del estado nutricional de gestantes universitarias, UNEMI 2018:}

resultados del plan piloto

Vol. 3, núm. 1., (2019)

Mariela Libelly Lozada Meza; Lorena Paola Ramírez Morán; Edwin Hernán Alvarado Chicaíza;

Carol Johana Cajas Bejarano

evaluación del estado nutricional en gestantes, que es una estrategia de atención primaria en salud (APS) que permite identificar tempranamente problemas de malnutrición sea por el déficit o exceso alimentario.

\section{Bibliografía.}

Fescina , R., De Mucio, B., Martínez , G., Alemán , A., Sosa, C., Mainero , L., \& Rubino , M. (2011). VIGILANCIA DEL CRECIMIENTO FETAL (2 ed.). Montovideo: Centro Latinoamericano de Perinatología / Salud de la Mujer y Reproductiva. Obtenido de https://www.paho.org/clap/index.php?option=com_docman\&view=download\&category_ slug=salud-de-mujer-reproductiva-materna-y-perinatal\&alias=229-vigilancia-delcrecimiento-fetal-manual-de-autoinstruccion-1\&Itemid=219\&lang=es

Goni, L., Martínez, A., Santiago, S., \& Cuervo, M. (2013). Validación de una encuesta para evaluar el estado nutricional y los estilos de vida en las etapas preconcepcional, embarazo y lactancia. Revista Española de Nutrición Comunitaria, 105-113.

Grandi, C., Luchtenberg, G., \& Sola, H. (2007). Evaluación nutricional durante el embarazo. Nuevo estándar. Revista Medicina, 1-8.

INCAP. (2012). Instituto de Nutrición de Centro América y Panamá. Obtenido de http://www.incap.int/index.php/es/publicaciones/doc_view/80-tabla-de-composicion-dealimentos-de-centroamerica

MSP. (2000). Ministerio de Salud Pública. Dirección Nacional de Nutrición PANN.

MSP. (2012). Ministerio de Salud Pública Ecuador. Obtenido de https://aplicaciones.msp.gob.ec/salud/archivosdigitales/documentosDirecciones/dnn/archi vos/S\%C3\%ADntesis\%20de\%20las\%20normas.pdf

MSP. (2014). Ministerio de Salud Pública Ecuador. Obtenido de http://www.saludzona1.gob.ec/cz1/images/PROGRAMAS/GUIASCLINICAS/2014/GPC \%20ALIMENTACION\%20Y\%20NUTRICIN\%20MUJER\%20EMB.pdf

MSP. (2016). Ministerio de Salud Pública Ecuador. Obtenido de https://www.salud.gob.ec/wpcontent/uploads/2016/09/ESTRATEGIA-REDUCCION-MUERTE-MATERNA.pdf

MSP. (2017). Ministerio de Salud Pública. Obtenido de https://www.inclusion.gob.ec/wpcontent/uploads/2018/07/GUIA-MATERNIDAD-Y-PRIMERA-INFANCIA-JUNIO-

CON-OBS.pdf 


\section{Evaluación del estado nutricional de gestantes universitarias, UNEMI 2018: resultados del plan piloto}

Vol. 3, núm. 1., (2019)

Mariela Libelly Lozada Meza; Lorena Paola Ramírez Morán; Edwin Hernán Alvarado Chicaíza; Carol Johana Cajas Bejarano

Rached, I. (2015). Evaluación nutricional de la embarazada. Centro de Atención Nutricional Infantil Antímano, 65, 1.

Suverza, A., \& Haua, K. (2010). El ABCD de la evaluación del estado de nutrición. Mexico: MC Graw Hill.

Torres, A. (2016). Repositorio digital. Escuela Superior Politécnica de Chimborazo. Obtenido de http://dspace.espoch.edu.ec/bitstream/123456789/7594/1/20T00919.pdf

UBA. (2016). Respositorio digital. Universidad de Buenos Aires. Obtenido de www.fmed.uba.ar/depto/nutrievaluacion/evaluacion2016.pdf

Worthington. (2012). Nutrición durante el embarazo y la lactancia. Nutrición y dietoterapia. Nutrición y dietoterapia, 153. 